\title{
Solution of Differential Equations using Exponential of a Matrix
}

\author{
Jervin Zen Lobo ${ }^{1}$, Terence Johnson ${ }^{2}$ \\ ${ }^{1}$ (Basic Sciences \& Humanities Department, Agnel Institute of Technology and Design, Goa University, India) \\ ${ }^{2}$ (Computer Engineering Department, Agnel Institute of Technology and Design, Goa University, India)
}

\begin{abstract}
Our main purpose in this project is to help reader find a clear and glaring relationship between linear algebra and differential equations, such that the applications of the former may solve the system of the latter using exponential of a matrix. Applications to linear differential equations on account of eigen values and eigenvectors, diagonalization of $n$-square matrix using computation of an exponential of a matrix using results and ideas from elementary studies form the core study of our project.

Keywords : matrix,fundamental matrix, ordinary differential equations, systems of ordinary differential equations, eigenvalues and eigenvectors of a matrix, diagonalisation of a matrix, nilpotent matrix, exponential of a matrix
\end{abstract}

\section{Introduction}

The study of Ordinary Differential Equation plays an important role in our life. Some applications include study of growth of microorganisms, population, decay of radiation, etc. Ordinary Differential equations is also used in medicine. Solving a first order Ordinary Differential Equation of first degree could be elementary as we have many ways of doing so - the Ordinary Differential Equation could be linear, homogenous; or we could solve it finding suitable integrating factor to make it exact, etc. In solving a second order non-homogenous differential equation, we have many methods namely: method of undetermined coefficient also called method of judicial guessing, method of variation of parameters, Inverse D-opertor method, etc. The homogenous part can well easily be solved by finding the roots of the auxiliary equation. However, as the order of the Ordinary Differential Equation goes higher, it becomes more tedious to solve the homogenous/non-homogenous part. In such cases, we reduce the $\mathrm{n}^{\text {th }}$ order Ordinary Differential Equation into a system of $\mathrm{n}$ first order Linear Differential Equation.

\section{Main Idea Of The Proposed Solution}

In this paper we propose to solve a system of first order linear differential equations using techniques of linear algebra. The innovative part of our paper is the use of exponential of a matrix to find the required solution of the system.

We know for any matrix $\mathrm{A}, e^{A}=I+A+\frac{A^{2}}{2 !}+\frac{A^{3}}{3 !}+\ldots$

If $\mathrm{A}$ is nilpotent of degree $\mathrm{n}$, the infinite sum above terminates at some point. In our paper, we use exponential of matrix in following manner:

Step 1-Write the given system in matrix form. Call the state transition matrix as A.

Step 2 - Find eigenvalues and eigenvectors of A. Form the matrix P whose columns are eigenvectors of A.

Step 3 - Compute $\mathrm{P}^{-1} \mathrm{AP}$ which will be diagonal. For example $\mathrm{P}^{-1} \mathrm{AP}=\operatorname{diag}\left(\alpha_{1} \beta_{1} \gamma\right)$.

Step $4-$ Write $A=P \operatorname{diag}\left(\alpha_{1} \beta_{1} \gamma\right) \mathrm{p}^{-1}$. Hence $\mathrm{t} A=\operatorname{Pdiag}\left(\operatorname{t} \alpha_{1} t \beta_{1} t \gamma\right) \mathrm{p}^{-1}$. Finally $\mathrm{e}^{\mathrm{tA}}=\operatorname{Pdiag}\left(\mathrm{e}^{\mathrm{t} \alpha} \mathrm{e}^{\mathrm{t} \beta} \mathrm{e}^{\mathrm{t} \gamma}\right) \mathrm{p}^{-1}$

Step 5 - Using result that general solution of $x^{1}=A x$ is $x(t)=e^{t A} C$. The solution is $e^{t A} \cdot C ; e^{t A}$ being obtained from step 4

\section{Solution of Differential Equations using Exponential of a Matrix}

Theorem: A matrix solution ' $\Phi(\mathrm{t})^{\prime}$ ' of $\Phi$ ' $=\mathrm{A}(\mathrm{t}) \Phi$ is a fundamental matrix of $\mathrm{x}^{\prime}=\mathrm{A}(\mathrm{t}) \mathrm{x}$ iff $\mathrm{w}(\mathrm{t}) \neq 0$ for $\mathrm{t} \square$ $\left(\mathrm{r}_{1}, \mathrm{r}_{2}\right)$.

Proof: Let $\Phi(\mathrm{t})$ be a fundamental matrix of $\mathrm{x}^{\prime}=\mathrm{A}(\mathrm{t}) \mathrm{x}$ with column vectors $\varnothing 1, \varnothing 2 \ldots \ldots \varnothing \mathrm{n}$. 
Let $\varnothing(\mathrm{t})$ be a solution of $\mathrm{x}^{\prime}=\mathrm{A}(\mathrm{t}) \mathrm{x}$. then $\phi(t)=\sum_{j=1}^{n} c_{j} \phi_{j}(t)$ for some constants $\mathrm{c}_{1}, \mathrm{c}_{2}, \ldots . . \mathrm{c}_{\mathrm{n}}$ not all zero.

By uniqueness of a solution $c_{j}$ 's are all unique.

If $\mathrm{c}=\left(\mathrm{c}_{1}, \mathrm{c}_{2}, \ldots \ldots . \mathrm{cn}\right)$ then $\varnothing(\mathrm{t})=\Phi(\mathrm{t}) \mathrm{c}$

For any to $\square\left(\mathrm{r}_{1}, \mathrm{r}_{2}\right)$ above relation represents $n$-linear algebraic equations in the $\mathrm{n}$-unknowns $\mathrm{c}_{1}, \mathrm{c}_{2}, \ldots \ldots \ldots \mathrm{cn}$ and has unique solution.

$\therefore$ Det $\Phi(\mathrm{t}) \neq 0 \Rightarrow \mathrm{W}(\mathrm{t}) \neq 0 \forall \mathrm{r}_{1}<\mathrm{t}<\mathrm{r}_{2}$

Let $\mathrm{W}(\mathrm{t}) \neq \mathrm{o} \forall \mathrm{t} \square\left(\mathrm{r}_{1}, \mathrm{r}_{2}\right)$

$\therefore \varnothing_{1}, \varnothing_{2} \ldots . . . \varnothing \mathrm{n}$ are linearly independent.

$\therefore \Phi(\mathrm{t})$ is a fundamental matrix.

Theorem: The general solution of $x^{\prime}=A x$ is $x(t)=$ et $A c$ where ' $c$ ' is an arbitrary constant vector. The solution of $x^{\prime}=A x$ with initial condition ' $x\left(t_{0}\right)=x_{0}$ ' is given by $x(t)=e^{(t-t) A}{ }_{0}^{A} x_{0}$.

Proof: Let $\mathrm{x}(\mathrm{t})$ be any solution of $\mathrm{x}^{\prime}=\mathrm{Ax}$

Define $u(t)=e^{(-t A)} x(t)=0$

$\therefore \mathrm{u}^{\prime}(\mathrm{t})=\mathrm{e}^{-\mathrm{tA}} \mathrm{x}^{\prime}(\mathrm{t})-\mathrm{A} \mathrm{e}^{(-\mathrm{tA})} \mathrm{x}(\mathrm{t})=0$

$\therefore \mathrm{u}(\mathrm{t})=\mathrm{c}$

i.e. $x(t)=e^{t A} c$.

Now, $\mathrm{x}\left(\mathrm{t}_{0}\right)=\mathrm{e}_{0}^{\mathrm{t}} \mathrm{A} \mathrm{c}$

$\mathrm{x}_{0}=\mathrm{e}_{0}^{\mathrm{t} A \mathrm{c}}$

$\mathrm{c}=\mathrm{e}^{-\mathrm{t}}{ }_{0}^{\mathrm{A}} \mathrm{x}_{0}$

$\therefore \mathrm{x}(\mathrm{t})=\mathrm{e}^{\mathrm{tA}} \mathrm{e}_{0}^{-\mathrm{t} A} \mathrm{x}_{0}$

$=\mathrm{e}_{0}^{(\mathrm{t}-\mathrm{t}) \mathrm{A}} \mathrm{x}_{0}$

$\Phi(\mathrm{t})=\mathrm{et} \mathrm{A}$ is the fundamental matrix of $\mathrm{x}^{\prime}=\mathrm{Ax}$, as

$\Phi^{\prime}(\mathrm{t})=\frac{d}{d t}\left(e^{t A}\right)$

$=A e^{t A}$

$=\mathrm{A} \Phi(\mathrm{t})$

Variation of Constant Formula:

Any solution of $\mathrm{x}^{\prime}(\mathrm{t})=\mathrm{A}(\mathrm{t}) \mathrm{x}(\mathrm{t})+\mathrm{B}(\mathrm{t})$ such that $\mathrm{x}\left(\mathrm{t}_{0}\right)=\mathrm{x}_{0}$ is given by,

$\mathrm{x}(\mathrm{t})=\Phi(t) x_{0}+\Phi^{-1}(s) B(s) d s$ where ' $\Phi(t)$ ' is a fundamental matrix of

$\mathrm{x}^{\prime}(\mathrm{t})=\mathrm{A}(\mathrm{t}) \mathrm{x}(\mathrm{t})$ such that $\Phi\left(t_{0}\right)=\mathrm{I}$.

Proof: Let $\mathrm{x}(\mathrm{t})=\Phi(t) \mathrm{C}(\mathrm{t})$, where $\mathrm{C}(\mathrm{t})$ is an unknown vector on $\left(r_{1}, r_{2}\right)$.

$$
\begin{aligned}
& \therefore \mathrm{x}\left(\mathrm{t}_{0}\right)=\Phi\left(t_{0}\right) \mathrm{C}\left(\mathrm{t}_{0}\right) \\
& \Rightarrow C\left(t_{0}\right)=x_{0} .
\end{aligned}
$$




$$
\begin{gathered}
\therefore x^{\prime}(t)=\Phi(t) C^{\prime}(t)+A(t) \Phi(t) C(t) \\
\text { i.e. } A(t) x(t)+B(t)=\Phi(t) C^{\prime}(t)+A(t) \Phi(t) C(t) \\
\text { i.e. } B(t)=\Phi(t) C^{\prime}(t) . \\
\therefore C^{\prime}(t)=\Phi^{-1}(t) B(t) . \\
\therefore \int_{t_{0}}^{t} C^{\prime}(s) d s=\int_{t_{0}}^{t} \Phi^{-1}(s) B(s) d s \\
\text { i.e.C(t)-C(t) } \left.t_{0}\right)=\int_{t_{0}}^{t} \Phi^{-1}(s) B(s) d s . \\
\therefore C(t)=x_{0}+\int_{t_{0}}^{t} \Phi^{-1}(s) B(s) d s \\
\therefore x(t)=\Phi(t) x_{0}+\int_{t_{0}}^{t} \Phi(t) \Phi^{-1}(s) B(s) d s .
\end{gathered}
$$

\section{Experimental Results}

Solve the following system of linear first order O.E.E.:

$$
\begin{aligned}
& \frac{d x}{d t}=x-y+4 z \\
& \frac{d y}{d t}=3 x+2 y-z \\
& \frac{d z}{d t}=2 x+y-z
\end{aligned}
$$

Soln: The given system can be put in the form.

$$
X^{\prime}=A X, \text { where } A=\left[\begin{array}{ccc}
1 & -1 & 4 \\
3 & 2 & -1 \\
2 & 1 & -1
\end{array}\right]
$$

The characteristic polynomial of matrix ' $A$ ' is

$$
\begin{aligned}
P(\lambda)=|A-\lambda I| & =\left|\begin{array}{ccc}
1-\lambda & -1 & 4 \\
3 & 2-\lambda & -1 \\
2 & 1 & -1-\lambda
\end{array}\right| \\
& =(1-\lambda)(\lambda-3)(\lambda+2)
\end{aligned}
$$

$\therefore$ The eigenvalues of ' $A$ ' are $\lambda_{1}=1, \lambda_{2}=3$ and $\lambda_{3}=-2$

Put $\lambda_{1}=1$ in $\left(\mathrm{A}-\lambda_{1}\right) \mathrm{V}=0$ we get,

$$
\left[\begin{array}{ccc}
0 & -1 & 4 \\
3 & 1 & -1 \\
2 & 1 & -2
\end{array}\right]\left[\begin{array}{l}
v_{1} \\
v_{2} \\
v_{3}
\end{array}\right]=\left[\begin{array}{l}
0 \\
0 \\
0
\end{array}\right]
$$


$\mathrm{R}_{1} \leftrightarrow \mathrm{R}_{3}$

i.e. $\left[\begin{array}{ccc}2 & 1 & -2 \\ 3 & 1 & -1 \\ 0 & -1 & 4\end{array}\right]\left[\begin{array}{l}v_{1} \\ v_{2} \\ v_{3}\end{array}\right]=\left[\begin{array}{l}0 \\ 0 \\ 0\end{array}\right]$

$\mathrm{R}_{1} \rightarrow \mathrm{R}_{1} / 2$

i.e. $\left[\begin{array}{ccc}1 & 1 / 2 & -1 \\ 3 & 1 & -1 \\ 0 & -1 & 4\end{array}\right]\left[\begin{array}{l}v_{1} \\ v_{2} \\ v_{3}\end{array}\right]=\left[\begin{array}{l}0 \\ 0 \\ 0\end{array}\right]$

$\mathrm{R}_{2} \rightarrow \mathrm{R}_{2}-3 \mathrm{R}_{1}$

i.e. $\left[\begin{array}{ccc}1 & 1 / 2 & -1 \\ 0 & -1 / 2 & 2 \\ 0 & -1 & 4\end{array}\right]\left[\begin{array}{l}v_{1} \\ v_{2} \\ v_{3}\end{array}\right]=\left[\begin{array}{l}0 \\ 0 \\ 0\end{array}\right]$

$\mathrm{R}_{2} \rightarrow \mathrm{R}_{2}-3 \mathrm{R}_{1}$

i.e. $\left[\begin{array}{ccc}1 & 1 / 2 & -1 \\ 3 & 1 & -1 \\ 0 & -1 & 4\end{array}\right]\left[\begin{array}{l}v_{1} \\ v_{2} \\ v_{3}\end{array}\right]=\left[\begin{array}{l}0 \\ 0 \\ 0\end{array}\right]$

$\mathrm{R}_{2} \rightarrow \mathrm{R}_{2}-3 \mathrm{R}_{1}$

i.e. $\left[\begin{array}{ccc}1 & 1 / 2 & -1 \\ 0 & -1 / 2 & 2 \\ 0 & -1 & 4\end{array}\right]\left[\begin{array}{l}v_{1} \\ v_{2} \\ v_{3}\end{array}\right]=\left[\begin{array}{l}0 \\ 0 \\ 0\end{array}\right]$

$R_{1} \rightarrow R 1-\frac{1}{2} R_{2}, R_{3} \rightarrow R_{3}+\frac{1}{2} R 2$

i.e. $\left[\begin{array}{ccc}1 & 0 & 1 \\ 0 & 1 & -4 \\ 0 & 0 & 0\end{array}\right]\left[\begin{array}{l}v_{1} \\ v_{2} \\ v_{3}\end{array}\right]=\left[\begin{array}{l}0 \\ 0 \\ 0\end{array}\right]$

$\therefore \mathrm{v} 1+\mathrm{v} 3=0$ and $\mathrm{v} 2-4 \mathrm{v} 3=0$

Choose $\mathrm{v}_{3}=1 \Rightarrow \mathrm{v}_{1}=-1, \mathrm{v}_{2}=4$.

$\therefore V_{1}=\left[\begin{array}{c}-1 \\ 4 \\ 1\end{array}\right]$ is an eigen vector of 'A' corresponding to the eigen value $\lambda_{1}=1$

Similarly, $V_{2}=\left[\begin{array}{l}1 \\ 2 \\ 1\end{array}\right]$ is an eigen vector of 'A' corresponding to the eigen value $\lambda 2=3$ and $V_{3}=\left[\begin{array}{c}-1 \\ 1 \\ 1\end{array}\right]$ is an eigen vector of ' $A$ ' corresponding to the eigen value $\lambda_{3}=-2$. 
Let $P=\left[\begin{array}{ccc}-1 & 1 & -1 \\ 4 & 2 & 1 \\ 1 & 1 & 1\end{array}\right]$ Then, $P^{-1}=\frac{1}{6}\left[\begin{array}{ccc}-1 & 2 & -3 \\ 3 & 0 & 3 \\ -2 & -2 & 6\end{array}\right]$

Now, $\mathrm{P}^{-1} \mathrm{AP}=\frac{1}{6}\left[\begin{array}{ccc}-1 & 2 & -3 \\ 3 & 0 & 3 \\ -2 & -2 & 6\end{array}\right]\left[\begin{array}{ccc}1 & -1 & 4 \\ 3 & 2 & -1 \\ 2 & 1 & -1\end{array}\right]\left[\begin{array}{ccc}-1 & 1 & -1 \\ 4 & 2 & 1 \\ 1 & 1 & 1\end{array}\right]$

$$
=\left[\begin{array}{ccc}
1 & 0 & 0 \\
0 & 3 & 0 \\
0 & 0 & -2
\end{array}\right]=\operatorname{diag}(1,3,-2)
$$

$\therefore \mathrm{P}-1 \mathrm{AP}=\operatorname{diag}(1,3,-2)$

$\therefore \mathrm{A}=\mathrm{P} \operatorname{diag}(1,3,-2) \mathrm{P}^{-1}$

$\therefore \mathrm{tA}=\mathrm{P}$ diag $(\mathrm{t}, 3 \mathrm{t},-2 \mathrm{t}) \mathrm{P}^{-1}$

$\therefore \mathrm{e}^{\mathrm{tA}}=\mathrm{P} \operatorname{diag}\left(\mathrm{e}^{\mathrm{t}}, \mathrm{e}^{3 \mathrm{t}}, \mathrm{e}^{-2 \mathrm{t}}\right) \mathrm{P}^{-1}$

$=\frac{1}{6}\left[\begin{array}{ccc}-1 & 1 & -1 \\ 4 & 2 & 1 \\ 1 & 1 & 1\end{array}\right]\left[\begin{array}{ccc}e^{t} & 0 & 0 \\ 0 & e^{3 t} & 0 \\ 0 & 0 & e^{-2 t}\end{array}\right]\left[\begin{array}{ccc}-1 & 2 & -3 \\ 3 & 0 & 3 \\ -2 & -2 & 6\end{array}\right]$

$=\frac{1}{6}\left[\begin{array}{ccc}e^{t}+3 e^{3 t}+2 e^{-2 t} & -2 e^{t}+2 e^{-2 t} & 3 e^{t}+3 e^{3 t}-6 e^{-2 t} \\ 4 e^{-t}+6 e^{3 t}-2 e^{-2 t} & 8 e^{t}-2 e^{-2 t} & -12 e^{t}+6 e^{3 t}+6 e^{-2 t} \\ -e^{t}+3 e^{3 t}-2 e^{-2 t} & 2 e^{t}-2 e^{-2 t} & -3 e^{t}+3 e^{3 t}+6 e^{-2 t}\end{array}\right]$

$\therefore$ the general solution of the given system is,

$\mathrm{X}=\mathrm{e}^{\mathrm{tA}} \mathrm{C}$.

i.e. $\left[\begin{array}{l}x \\ y \\ z\end{array}\right]=\frac{1}{6}\left[\begin{array}{ccc}e^{t}+3 e^{3 t}+2 e^{-2 t} & -2 e^{t}+2 e^{-2 t} & 3 e^{t}+3 e^{3 t}-6 e^{-2 t} \\ 4 e^{-t}+6 e^{3 t}-2 e^{-2 t} & 8 e^{t}-2 e^{-2 t} & -12 e^{t}+6 e^{3 t}+6 e^{-2 t} \\ -e^{t}+3 e^{3 t}-2 e^{-2 t} & 2 e^{t}-2 e^{-2 t} & -3 e^{t}+3 e^{3 t}+6 e^{-2 t}\end{array}\right]\left[\begin{array}{c}c_{1} \\ c_{2} \\ c_{3}\end{array}\right]$

where $c_{1}, c_{2}, c_{3}$ are arbitrary constants.

\section{Conclusion}

Mathematics is one of those few fields of study in which a given problem can be tackled using more than one approach. In our paper, we have solved systems of differential equations using techniques of linear algebra by exponential of matrix method. However, there are certain limitations, one of which is the inability to find the exponential of our matrix if the matrix is non-diagonalizable or the given matrix is not nilpotent or if the given matrix possesses complex eigen values. 


\section{References}

[1] Cleve Moler, Charles Van Loan, Nineteen dubious ways to compute Exponential of a matrix, Twenty five years later, Siam Review s Vol 45 No 1 pp. 3-000 (2003 Society for industrial and Applied Mathematics

[2] J. Gallier and D. Xu, Computing Exponentials of skew-symmetric matrices and logarithms of orthogonal matrices (International journal of robotics and automation Vol 17 no. 4 2002)

[3] Su-Jing Wang,Cheng, Cheng jia, Hui-ling Chen, Chun Guang Zhou, college of computer science and technology, Jilin university, Chang Chun 130012 P.R china.

[4] M. Arioli, B. Cobenotti and C. Fassino, The Pade method for computing metrix exponential, Lin. Alg. Application, 240 (1996) pp. $111-130$

[5] R.B Sidje, explosive, software package for computing matrix exponential. ACM Trans Math software 24 (1998) pp. 130-156.

[6] G.F. Simmons, Differential equations with applications and historical notes (Tata McGraw hill publishing company Ltd)

[7] E.A. Coddington, A textbook on ordinary differential equations (Prentice Hall Publications)

[8] Krishnamurthy and others, An introduction to linear algebra (Affiliated East-West Press Pvt. Ltd)

[9] Shantinarayan, A textbook on matrices (S. Chand and company Ltd.)

[10] K.B. Datta, Matrix and linear algebra (Prentice Hall of India Pvt. Ltd.)

[11] M. Rama MohanaRao, Ordinary differential equations, Theory and applications (Affiliated East-West Press Pvt. Ltd) 